Relations industrielles

Industrial Relations

\title{
European Coal Mining Union: Structure and Function, by Frederic Meyers, Institute of Industrial Relations, University of California, Los Angeles, 1961.
}

\section{Laurent Bélanger}

Volume 16, numéro 3, juillet 1961

URI : https://id.erudit.org/iderudit/1021783ar

DOI : https://doi.org/10.7202/1021783ar

Aller au sommaire du numéro

Éditeur(s)

Département des relations industrielles de l’Université Laval

ISSN

0034-379X (imprimé)

1703-8138 (numérique)

Découvrir la revue

Citer ce compte rendu

Bélanger, L. (1961). Compte rendu de [European Coal Mining Union: Structure and Function, by Frederic Meyers, Institute of Industrial Relations, University of California, Los Angeles, 1961.] Relations industrielles / Industrial Relations, 16(3), 363-366. https://doi.org/10.7202/1021783ar

Tous droits réservés (C Département des relations industrielles de l’Université Laval, 1961
Ce document est protégé par la loi sur le droit d'auteur. L'utilisation des services d'Érudit (y compris la reproduction) est assujettie à sa politique d'utilisation que vous pouvez consulter en ligne.

https://apropos.erudit.org/fr/usagers/politique-dutilisation/ 
lation qui restreigne indùment le droit de grève et amène l'intervention de l'Etat aux premiers stages d'un conflit. «L'intérêt public » est beaucoup moins souvent en cause qu'on ne le croit.

\section{L.}

Famille, industrialisation, logement, par Andrée Michel, Centre National de la Recherche Scientifique, 13 Quai Anatole France, Paris VII, 1959.

Pour ceux qui s'intéressent à la sociologie familiale, en particulier, aux modifications dans les structures et les systèmes de valeurs familiaux par suite de la révolution industrielle, voici un ouvrage élaboré sur le sujet.

L'étude porte sur 276 ménages habitant les hôtels meublés à Paris et dans la Seine. Un premier chapitre donne une description de l'habitat et des conditions économiques.

Les transformations au sein de la vie familiale pour s'ajuster au conditionnement externe du système industriel accusent des différences suivant les types de famille: industrielle française, semipatriarchale espagnole, patriarchale algérienne. La structure économique d'un cadre rural archaïque et la structure juridique établissaient un mode de transmission de la propriété dans la famille ancienne patriarchale. Les membres tiraient leur identité du groupe familial. Chacun avait un statut et un rôle fixes. La hiérarchie consacrait la supériorité des parents sur les enfants, celle des sexes fut présentée comme de droit divin, la morale sexuelle était respectée, le mariage était considéré comme un sacrement, les liens de parenté s'étendaient presque à l'infini, la sécurité était assurée totalement par la famille ou le voisinage. Dans l'ensemble, l'organisation interne de la famille était basée sur la hiérarchie, le conformisme et la répression. La famille industrielle présente un modèle différent. Elle tire son mode d'existence du salariat, possède peu de biens. La tendance à l'égalitarisme règne dans les relations entre époux, entre parents et enfants. L'infériorité de la femme disparaît si on considère le rôle qu'elle joue dans l'administration des biens. La liberté de choix du conjoint pour les jeunes, les loisirs à l'extérieur du ménage, l'interchangeabilité des rôles entre l'homme et la femme diminuent l'autorité du «chef de famille ». On considère les institutions (mariage, religion) comme étant au service de la personne et non l'inverse.

Des transformations économiques accompagnent le passage de la famille ancienne à la famille conjugale: « partage par la famille de ses rôles traditionnels avec des tiers (organismes de sécurité sociale, de loisirs) indépendance de la personne par rapport à un rôle et un statut préétablis et fixes, tendance à l'identification sociale avec un rôle extérieur au groupe familial, concurrence des valeurs économiques ». Cette évolution enlève le caractère sacré aux rites relatifs à la naissance (idéal de la famille prolifique), à l'adolescence, au mariage et à la mort.

Au cours de l'adaptation de la vie familiale aux conditionnements externes engendrés par la guerre et la révolution industrielle, une conception de la famille ancienne a survécu. Elle diffère cependant selon le type de famille étudié. L’auteur signale un « retard culturel » qui consiste en la « dysharmonie entre les fonctions économiques de la famille d'aujourd'hui et l'idée ancienne que l'on s'en fait encore. Cette étude amène l'auteur à préciser le concept de «famille». \& Dans sa forme la plus complète, la famille conjugale est constituée par le père, la mère, les enfants, mais elle peut aussi se reduire au couple sans enfants, à un adulte ayant un ou plusieurs enfants.

Dans la conclusion, l'auteur met en exergue les facteurs qui paraissent agir le plus sur la structure de la famille:

a) la structure de la société globale (développement technologique, division du travail, mode de propriété).

b) l'intégration des familles aux classes sociales ou aux multiples groupements.

c) le logement.

LAURent Bélanger

European Coal Mining Union: Structure and Function, by Frederic Meyers, Institute of Industrial Relations, University of California, Los Angeles, 1961. 
Cette étude se veut à la fois descriptive et comparative. L'auteur se propose d'analyser la structure et les fonctions du syndicalisme dans les mines de charbon européennes. L'industrie du charbon en France, Belgique, Allemagne de l'Ouest et en Grande-Bretagne constitue l'unité de base de son analyse. Cette industrie comporte un haut degré d'homogénéité sur le plan des techniques d'exploitation et des conditions de travail. Cependant, les formes de propriété diffèrent avec les pays. En France et en Grande-Bretagne, les mines de charbon sont nationalisées. En Allemagne et en Belgique, l'industrie minière entre les mains de capitaux privés.

Au plan structurel - C'est d'abord l'exposé de la hiérarchie des institutions: unions locales, organisations intermédiaires, unions nationales, fédérations.

En Allemagne, l'Industriegewerkshaft Bergau (IGB) demeure la seule centrale syndicale importante. Quoiqu'elle se veuille neutraliste sur le plan idéologique, le pluralisme existe en fait. Devant la promotion d'objectifs socialistes et sociaux démocrates, les leaders catholiques se croient défavorisés et veulent rétablir une centrale syndicale chrétienne.

En Grande-Bretagne, la National Union of Mineworkers (NUM) constitue la principale union minière. Elle est affiliée au Trade-Union Congress. Le pluralisme syndical ne se rencontre pas sur une base idéologique. La structure du syndicalisme anglais est un chevauchement d'union de métiers, unions amalgamées, industrielles ou générales.

En Belgique, le pluralisme idéologique et structurel prévaut. La F.G.T.B. avec son orientation socialiste et la C.S.C. (chrétienne) constituent les deux centrales syndicales nationales. La Centrale syndicale des Travailleurs des Mines de Belgique est affiliée à la F.G.T.B.; la Centrale des Francs Mineurs, à la C.S.C.

En France, le même phénomène existe. Trois centrales représentant les travailleurs: la C.G.T. (communiste), la C.G.T.-Force-Ouvrière et la C.F.T.C. (chrétienne). A chacune de ces centrales se trouvent affiliées les unions minières industrielles.

L'auteur décrit ensuite les organes du gouvernement interne des unions. Les noms diffèrent d'un pays à l'autre, mais la hiérarchie des organismes demeure à peu près la même. L'élection ou la nomination des représentants ouvriers au sein des comités varient avec les pays étudiés.

Au plan fonctionnel - En général, le syndicalisme européen s'acquitte de deux fonctions principales: la négociation collective et l'action politique.

Négociation collective - Dans chaque pays étudié, l'existence d'institutions fournissant une participation des travailleurs à la gestion et l'extension d'une détermination légale des conditions de travail conditionnent la sphère des négociations.

L'idéologie de la possession et du contrôle de l'industrie par les travailleurs explique l'existence des \& work concils ». L'idéologie sociale chrétienne, moins radicale, met de l'avant le principe de la cogestion, i.e. la participation conjointe des représentants des travailleurs et du capital dans la direction de l'entreprise.

Le rôle des «works concils» varie d'un pays à l'autre. En Angleterre, ce sont des comités consultatifs; alors qu'en Allemagne ces conseils ont un pouvoir de décision, cependant ils demeurent subordonnés à la négociation collective.

En France, la loi n'exige pas l'établissement de ces conseils. Les Charbonnages de France sont dirigés par une commission directoriale où siègent les membres représentant les groupes fonctionnels: les travailleurs, les consommateurs de charbon, les représentants de l'Etat.

La détermination légale des conditions de travail joue un rôle important en France et en Belgique. En Allemagne et Grande-Bretagne, cette détermination est laissée à la négociation.

L'accomplissement de la fonction de négociation et administration de la convention varie aussi avec les pays.

En France, le processus de négociation a un caractère politique et consultatif: les conditions d'emploi sont définies par des lois dont la principale est le Statut du Mineur. L'autorité directoriale demeure dans les mains de directeurs généraux, issus des rangs des ingénieurs miniers. La négociation se fait avec les hauts représentants des unions minoritaires, puisque la C.G.T. est exclue. La 
fonction administrative revient aux niveaux plus pas de la hiérarchie directoriale et des unions.

En Allemagne, les termes de l'accord concernant les conditions d'emploi sont contenus dans trois documents: une convention de base signée entre l'union et l'association d'employeurs de la région du Rurh, un deuxième accord signé pour les autres districts où un officier régional à plein temps prend charge des négociations et agit selon les directives de l'union nationale; enfin, un accord au niveau de l'établissement entre l'employeur et le «work concil». Le pouvoir réel et l'autorité en négociation sont exercés par le comité exécutif de l'union nationale.

En Grande-Bretagne, après la nationalisation, la discussion a lieu entre la National Union of Mineworkers et la National Coal Board. Un comité national de négociation est établi: the Joint National Negotiating Committee. Ce comité ne peut conclure un accord qui liera les parties. L'exécutif régional exerce une autorité formelle et effective en négociation.

En Belgique, la négociation collective se fait par l'intermédiaire de la Commission Nationale Mixte des Mines. Les décisions unanimes de la commission ont la force d'un accord. Le pouvoir de lier les parties ne s'exerce qu'après consultation des comités exécutifs des unions.

Dans chaque pays, la fonction administrative est exercée au niveau local. Les modalités et procédures de règlements de griefs diffèrent. La composition et les fonctions des organismes se chargeant de la sécurité industrielle font l'objet d'un chapitre complet. L'auteur consacre aussi un chapitre au contrôle des fonds syndicaux et des déclarations de grèves.

Action politique - L'accent sur les objectifs politiques et les liens qu'entretiennent les mouvements ouvriers varient selon les idéologies qui prônent un changement institutionnel. Le syndicalisme européen est considéré comme révolutionnaire, mais cette conception doit être qualifiée. Les idéologies socialiste, anarchosyndicaliste et communiste veulent en longue période le renversement des institutions ou l'abolition du régime capitaliste. Le mythe de la grève générale planne sans cesse sur les mouvements. Par contre, l'idéologie chrétien- ne pròne une réforme structurelle dans le sens d'une plus grande participation des travaileurs à la direction et d'une réduction des inégalités économiques et sociales.

La diversité sur le plan des objectifs entraîne des différences sur le plan des réalisations.

Dans trois des quatre pays, la GrandeBretagne exceptée, les constitutions imposent la neutralité politique. Cependant, les liens entre les unions et les partis politiques sont réels. La relation la plus étroite se trouve entre la C.G.T. et le parti communiste français. Les officiers régionaux et nationaux sont pour la plupart membres du parti. L'action politique au sein de la C.G.T. se fait sur une base individuelle. La structure du pouvoir est décentralisée puisque la réalisation des objectifs repose sur une action de masse. Force-Ouvrière demeure dans la tradition des unions françaises: celle d'obtenir des réformes de structure de base. La C.F.T.C. entretient des relations étroites avec le MRP.

En Belgique, la tentative de fusion des Syndicats Uniques, dominés par les communistes avec la F.G.T.B. a échoué. Le mouvement ouvrier chrétien a décliné toute proposition d'unité syndicale et adhére au principe du pluralisme idéologique.

En Allemagne, la coalition entre les unions chrétiennes et socialistes s'est opérée sur la base d'une neutralité politique. Le programme de l'union met de l'avant l'objectif de la nationalisation des mines. Cette coalition favorisa l'obtention de mesures législatives immédiates.

En Grande-Bretagne, des relations formelles existent entre le Trades-Union Congress et le parti travailliste. Le Congrès domine le parti. Les unions peuvent aider au financement du parti, avec la contribution obligatoire des membres.

La tentative d'expliciter les variables qui peuvent rendre compte de la diversité des fonctions, et partant, des structures, au sein du syndicalisme européen demeure le point culminant de l'étude comparative.

Les mouvements ouvriers européens ont un objectif ultime commun: celui d'améliorer le statut économique et social des travailleurs. L'auteur tente de démontrer que, même s'il y a homogénéité 
au plan de l'objectif ultime, il y a aussi une pluralité sur les plans structurel et idéologique.

La priorité accordée à l'action politique plutôt qu'à la négociation collective explique pour une part la centralisation ou la décentralisation du pouvoir au sein des mouvements. La forme de propriété de l'entreprise rend compte aussi de la centralisation du pouvoir. Nationalisée ou entre les mains de capitaux privés, la propriété demeure centralisée. Le pluralisme idéologique et structurel ne peut être expliqué par la nature de l'industrie, puisque l'idéal politique ne conduit pas nécessairement au pluralisme syndical. La Grande-Bretagne illustre ce fait. $\mathrm{La}$ variable décisive dans l'explication du pluralisme serait la suivante: «The crucial determinants of union structure is the view that a national labor movement or a national working class takes of its own role».

Cette analyse constitue un document important pour ceux qui étudient le syndicalisme avec l'approche structurellefonctionnelle ou encore pour ceux qui s'intéressent aux problèmes de démocratie syndicale.

\section{LAURENT BÉLANGER}

La vie quotidienne des familles ouvrières, par M. Chombart de Lauwe, Centre National de la Recherche Scientifique, Letouzey et Ané, GentillySeine, 1959.

Cette étude empirique sur la vie des familles ouvrières est un exposé des relations entre les conditions de vie de ces familles et leurs comportements sociaux, en particulier leur comportement alimentaire. L'interprétation des données tient compte toujours du milieu social, i.e. «de l'ensemble des facteurs sociaux se trouvant en relation avec les comportements et les personnalités des sujets et avec la forme et comportement des groupes 》.

L'orientation générale de la recherche est de dépasser la description de la situation et l'interprétation quantitative des rapports entre les deux grandes variables, pour découvrir les facteurs psychosociologiques qui sous-tendent les comportements sociaux. Ces facteurs sont les réactions des familles à l'endroit de leurs conditions de vie, leurs attitudes, leurs aspirations, leurs motivations, leurs coutumes, etc.

L'enquête, portant sur les travailleurs salariés et leur famille habitant les quartiers de Paris et de la banlieue, commen. ce en 1950. C'est la reprise économique après une récession. La conjoncture économique est un facteur dont il faut tenir compte dans l'interprétation des données puisque l'intérêt * tient au fait que les familles ouvrières sont le récepteur le plus sensible aux perturbations économiques et aux déséquilibres sociaux 》.

Pour préciser le concept de famille ouvrière, correspondant à classe ouvrière, l'auteur établit des catégories et souscatégories socio-professionnelles. L'interprétation des rapports entre conditions de vie et comportements montre les variations sensibles suivant la classe et les catégories professionnelles au sein de la classe ouvrière.

La variable «conditions de vie » comprend les éléments suivants: le travail de l'homme et ses conditions d'existence, le travail de la femme et l'équilibre du ménage, les conditions de logement et l'espace familial.

Au chapitre du travail de l'homme, l'auteur souligne l'opposition entre le milieu de travail et le milieu résidentiel: le premier est un milieu où l'activité est orienté vers la production, l'adaptation aux techniques nouvelles; le second est un milieu de consommation, où dominent les valeurs traditionnelles, les relations familiales et de voisinage. L'emploi, le métier et la vie syndicale ont des répercussions sur la vie familiale.

Le travail de la femme impose un rythme à la vie de famille. Ce dernier, en retour, influe sur le rendement dans l'entreprise. Ce travail modifie les rapports entre parents et enfants, les habitudes de consommation par l'apport d'un revenu accru.

L'étude sur les logements se centre sur les points suivants: ce qu'expriment les logements et conditionnements qu'ils imposent; ce que devraient être les logements. L'auteur arrive à la détermination d'un seuil critique au-dessous duquel les maladies physiques ou mentales peuvent apparaître. Il traite du décalage entre les désirs et les conditions existantes, des types de rapports sociaux entre familles ouvrières et familles bourgeoises. 